\title{
Propagation modeling in two transverse dimensions of the National Ignition Facility baseline performance
}

\author{
R.A. Sacks, W.W. Williams, M.A. Henesian, C.D. Orth, S.W. Haney, \\ J.B. Trenholme, J.M. Auerbach, J.K. Läẅson
}

This paper was prepared for submittal to the 2nd Annual International Conference on Solid State Lasers for Application to Inertial Confinement Fusion Paris, France

October 22-25, 1996

February 25, 1997

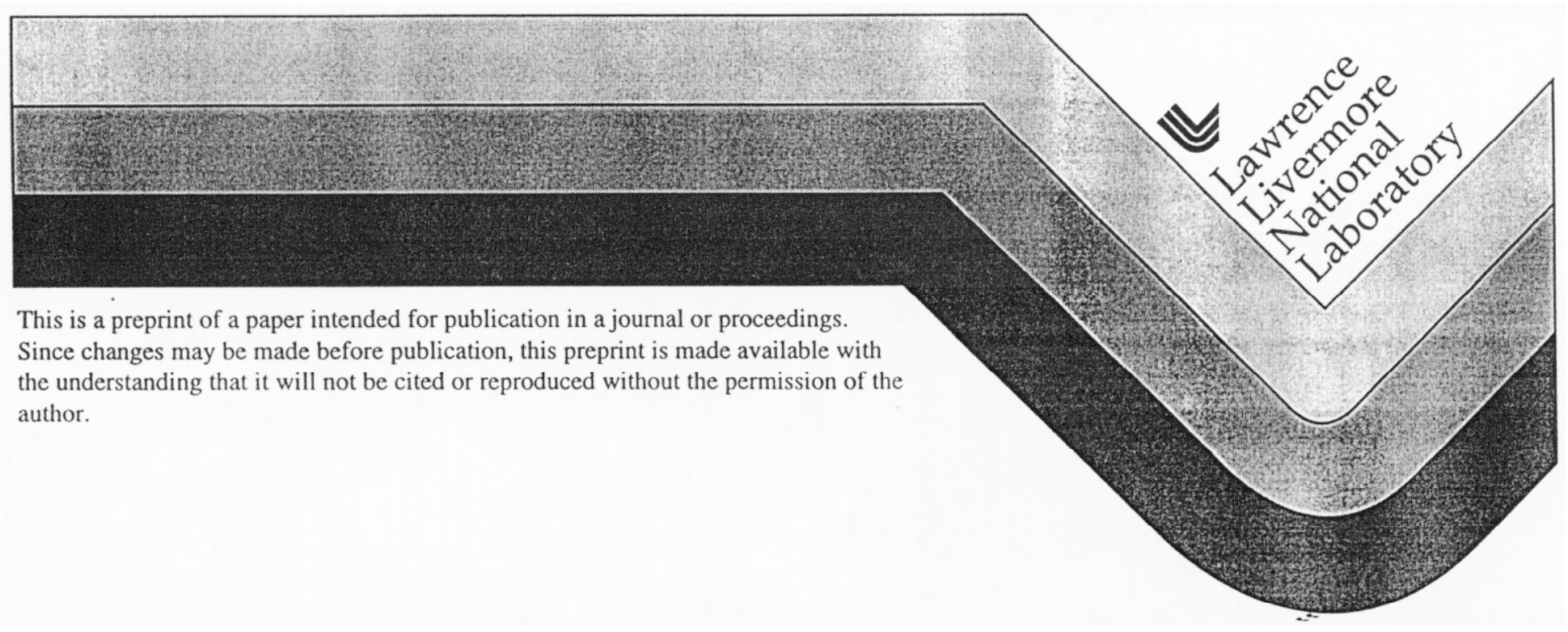




\section{DISCLAIMER}

This document was prepared as an account of work sponsored by an agency of the United States Government. Neither the United States Government nor the University of California nor any of their employees, makes any warranty, express or implied, or assumes any legal liability or responsibility for the accuracy, completeness, or usefulness of any information, apparatus, product, or process

disclosed, or represents that its use would not infringe privately owned rights. Reference herein to any specific commercial product, process, or service by trade name, trademark, manufacturer, or otherwise, does not necessarily constitute or imply its endorsement, recommendation, or favoring by the United States Government or the University of California. The views and opinions of authors expressed herein do not necessarily state or reflect those of the United States Government or the University of California, and shall not be used for advertising or product endorsement purposes. 


\title{
Propagation modeling in two transverse dimensions of the National Ignition Facility baseline performance
}

\author{
R. A. Sacks, W. W. Williams, M. A. Henesian, C. D. Orth, S. W. Haney, J. B. Trenholme, \\ J. M. Auerbach, and J. K. Lawson \\ Lawrence Livermore National Laboratory \\ P. O. Box 808 , L-490 \\ Livermore, CA $94551-9900$
}

Design optimization of the 1.8 MJ, $500 \mathrm{TW}$ National Ignition Facility (NIF) laser has proceeded with the use of a suite of new computational models. Cost-effectiveness of alternative fundamental architectures was considered using CHAINOP. A very fast, lumped-element energetics code, CHAINOP includes an extensive cost database, a runtime choice of optimization algorithm, and a set of heuristic rules for diffraction and nonlinear effects and for operational constraints. Its ability to flexibly consider many alternative configurations at a few seconds per chain made it the ideal "first-cut" tool for narrowing the investigation to the switched, multi-pass cavity architecture that was chosen.

Nonlinear-index effects-magnification of intensity modulation and generation of filamentary damage-were studied mainly, though not exclusively, with the Bespalov-Talinov code BTGAIN. Written originally by J. Trenholme and extensively modified and extended by P. A. Renard, this code calculates in the far-field the growth of single-mode perturbations on a planewave background beam due to the effects of the nonlinear refractive index. It includes FrantzNodvik saturated gain, the facility to input beam perturbations of arbitrary spectral content at each component, and a post-processor that can construct near-field beam statistics.

Detailed optimization and performance validation of the chosen NIF baseline were carried out using the Fourier propagation code PROP92, along with the 1D and 2D nonlinear frequency conversion codes THGXTZ001 and THG4D01. The use of PROP92 in its 1D mode to optimize the NIF design, as well as the physics and operation of the frequency conversion codes, is detailed elsewhere in this proceedings. Here we discuss the use of the 2D version of PROP92 to carry out detailed performance verification of the selected baseline design.

PROP92 is a full-featured Fourier-optics laser modeling, design, and optimization tool. The paraxial nonlinear Schrödinger equation (NLS) is solved using FFTs to move between the near field where nonlinear and gain effects are computed, and the far-field, where propagation is done. The Talinov transformation is used to remove average wavefront curvature from the stored field array, and a Lanczos option is included to relax the paraxial requirement. Gain saturation is treated in the Franz-Nodvik approximation, and nonlinear index effects are included by a splitstep phase advance algorithm. As is described elsewhere in this Proceedings, PROP92 has been interfaced with sophisticated systems analysis and optimization tools, and the resulting capability was used for extensive systems studies of NIF design parameters. ${ }^{1}$ 
Semi-analytic models of optical damage and filamentation have been included in PROP92. The damage model is based on the observation that the damage fluence for optical materials has been found ${ }^{2}$ to scale as

$$
\Phi_{D}=C \tau^{\beta}
$$

(1)

where $t$ is the full-width at half maximum of the nearly-Gaussian pulses that were used in the damage testing, and $C$ and $b$ are phenomenological constants. In an effort to apply this database to non-Gaussian pulses, J. Trenholme ${ }^{3}$ developed a diffusion-like model for damage. A damage integral

$$
D(t)=A \int_{0}^{\infty} \frac{I(t-s)}{s^{\beta}} d s
$$

(2)

is compared with the corresponding integral at the Gaussian-pulse damage limit

$$
D_{D}=\frac{A C}{\sqrt{\pi}}(4 \ln 2)^{\beta / 2} \max _{\xi} \int_{0}^{\infty} \frac{e^{-(\xi-s)^{2}}}{s^{\beta}} d s
$$

(3)

Any spatial grid-point for which the maximum over time of $D(t)$ is greater than $D_{D}$ is assumed to damage. PROP92 accumulates and reports the number of such grid points and the maximum over space and time of $D(t) / D_{D}$

PROP92's split-step treatment of nonlinear index effects has been shown ${ }^{4}$ to be capable of accurately tracking the self-focusing of intensity spikes up to the point where optical breakdown (not included in the model) would occur. Such calculations, however, must be done in 2D (filament collapse does not occur in fewer dimensions) and require high spatial resolution. To enable checking for the possibility of filamentation during less well-resolved (hence, faster) calculations, we have implemented two features that warn of threatening conditions. The first is based on the observation ${ }^{5}$ that intensity perturbations of the most unstable size and shape will collapse during propagation through a uniform nonlinear medium, in a distance such that $\mathrm{D} B_{0}=$ $g I_{0} \mathrm{D} z=2.3$, where $I_{0}$. is the maximum intensity at the peak of the initial perturbation, and $g=$ $n_{2} / n_{0} c e_{0}$ measures the material nonlinear susceptibility. By detecting and reporting the maximum over transverse position of the $\mathrm{D} B$ through the thickness of any given optic, PROP92 tracks the safety margin with respect to collapse in that optic. To allow for optical gain (which is also calculated by a split-step algorithm), the maximum $\mathrm{D} B(x, y)$, from the current $z$-position to the exit face of the optic, is re-calculated at each z-step. The second feature uses the fact that late in the process of collapse all perturbations evolve toward a shape in which the intensity scales as $\left(z_{c}-z\right)^{-4 / 3}$, where $z_{c}$ is the position of the singular collapse point. At each $z$-step through the slab, the maximum calculated irradiance is scaled by $\left[\mathrm{z}_{\mathrm{c}}\left(\mathrm{z}_{\mathrm{c}}-\mathrm{z}_{\text {exit }}\right)\right]^{4 / 3}$ to project a maximum anticipated self-focused irradiance in the slab. A warning message is printed if this projected irradiance exceeds a user-entered break-down irradiance. 
Using PROP92 operating in its 1D mode, some tens of thousands of variants on the basic 4pass switched-cavity architecture were examined for projected $3 w$ energy in each of the three NIF-prescribed pulse shapes, safety from optical damage and filamentation, and operational constraints such as injection energy, flashlamp size and voltage, and alignment tolerance. A detailed cost estimate was generated for each configuration, and a cost-optimized laser design that meets operating and safety margins with appropriate margin was selected. That design is shown in Figure 1. Detailed 2D calculations described below verify that this design will adequately fulfill the NIF mission.

In Figure 2 we show the transverse gain distribution assumed for these calculations, along with the gain measurements from the Beamlet chain. The NIF shape is derived from the Beamlet measurements with some smoothing and symmetrization, and an assumption that the ASEengendered edge droop can be cut in half by properly designed shaped reflectors. Note that because of the asymmetric pumping the end slabs have a significant gain tilt, and that this effect was retained.

Allowable imperfections in optical surfaces or nonuniformity of refractive indices will be specified for NIF based on the power spectral density (PSD) of the wavefront distortions. In PROP92, those wavefront distortions are represented as phase screens which, among other options, can be input numerically. Aberration files for these simulations were created by measuring the PSD of corresponding parts on Beamlet, and creating stochastic phase screens that match those PSDs. Figure 3 shows the PSD dependence on spatial frequency for the principal optical components in the NIF chain. The different curves on each plot are the result of collapsing the 2D measured PSD onto $1 \mathrm{D}$ in 6 different directions; the actual 2D phase screens were constructed using the full 2D PSD information. The roll-off at $1 \mathrm{~mm}^{-1}$ is a result of the finite resolution of the calculation. In Figure 4 we display the physical phase screens generated to represent one of the amplifier slabs. To avoid unphysical coherent addition, each slab is represented by a unique phase screen having the same PSD.

When the flashlamps fire to pump the slabs, they generate a prompt, asymmetric thermal load on the glass, leading to strain and hence to optical steering. Since this pump-induced distortion could potentially have a major effect on the obtainable spot size, it was important to include it in the simulation. The steering measured on Beamlet has been successfully modeled by a sequence of $\operatorname{codes}^{6}$ that 1) calculate the thermal loads from the profile of pump light entering the front and back slab surfaces, 2) from that calculate the thermo-mechanical strain in the glass, and 3 ) from that predict the position-dependent ray steering. As no reliable prediction of the incident light distribution was available for this work, it was assumed that it was quadratic in the horizontal direction, with one-half the curvature that would be necessary to give flat gain. The same opto-thermo-mechanical calculation procedure then led to the steering profiles shown in Figure 5. As the steering is dominated by horizontal dependence, vertical invariance was assumed. 
To achieve acceptably-smooth target-plane irradiance, NIF will utilize a combination of a quasi-continuous-height random phase plate (kinoform phase plate, or KPP) and ID or 2D smoothing by spectral dispersion (SSD). The KPP has been designed ${ }^{7}$ to yield a specified farfield spot envelope when a flat-phase beam is incident on it. Figure 5 also shows the phase screen predicted by that procedure and used in these simulations. No systematic modeling of SSD effects has yet been incorporated.

NIF is optimized to fulfill three primary missions: science-based stockpile stewardship (SBSS), indirect-drive inertial confinement fusion (ICF), and nuclear weapons effects testing (NWET). Direct-drive ICF will also be supported but did not play a major role in design decisions. In Figure 6, one sees call-outs describing the pulse length and format, and the energy, power, and spot-size requirements for each of these three missions. Also seen are the squarepulse performance curves predicted by PROP92 in 1D: the $1 w$ power vs. energy curve as limited by the constraint, the $\mathrm{D} B$ between spatial filters $\leq 1.8$, and the corresponding $3 w$ curve when those pulses are tripled. The latter curve is double-valued because at long pulse length the $1 w$ irradiance falls to values that convert poorly. Superimposed on the $3 w$ curve are the results of our three baseline $2 \mathrm{D}$ calculations. In all three cases, these show the predicted deliverable $3 w$ power and energy in the specified pulse format inside the specified spot at damage ratio of 1-i.e., just before damage is initiated. In all three cases, the performance requirement is exceeded.

As illustrated in Figure 7, our simulations consistently point to the exit face of the tripler as NIF's "fuse," the first element to damage as the power is increased. This conclusion must be softened by noting that at the resolution available here, no consideration was possible of the effects of small defects which could act as sources for filamentary growth, and that the conclusion is dependent on the assumed values for the damage fluence of the different components. Nevertheless, we believe that improvements in the $3 w$ damage threshold of the $\mathrm{KD} * \mathrm{P}$ crystals could pay one-for-one dividends in NIF's deliverable energy.

Figures 8 and 9 show the predicted near-field $1 w$ beam at the input to the doubler, and the far-field $3 w$ spot at the target plane for each of the three primary missions. The near-field contrast is the rms intensity variation divided by the average intensity for the central flat portion of the beam. There is some evidence from Beamlet measurements that once the contrast exceeds 0.1 , it grows rapidly with further irradiance increases. The far-field spots appear to be adequate for the mission requirements. The considerable high-frequency modulation will be smoothed by the imposition of SSD.

In summary, the performance of the NIF baseline design has been modeled in two transverse dimensions using the Fourier optics code PROP92 and the nonlinear harmonic conversion code THG4D01. The results obtained are in good agreement with those of the 1D versions of these codes which were used during the design optimization, yielding good confidence that a nearoptimal design has been chosen. We project that this design is able to fulfill NIF's three major mission specifications without component damage. Further modeling, including the effects of airpath turbulence, quasi-static thermal deformations, SSD, and sensitivity to misalignment and component tolerances is ongoing. 


\section{ACKNOWLEDGMENT}

This work was performed under the auspices of the U.S. Department of Energy by Lawrence Livermore National Laboratory under Contract No. W-7405-Eng-48.

\section{REFERENCES}

1. S. W. Haney, W. W. Williams, R. A. Sacks, C. D. Orth, J. M. Auerbach, J. K. Lawson, M. A. Henesian, K. S. Jancaitis, P. A. Renard, and J. B. Trenholme, "The Optimized NIF Laser System Based on Target Requirements," Second Annual International Conference on Solid State Lasers for Application to Inertial Confinement Fusion, Paris, France, October 22-25, 1996.

J. H. Campbell and F. Rainer, "Optical Glasses for High-Peak-Power Laser Applications," UCRL-JC-109255 (1992); Proceedings of the 1992 SPIE Conference, San Diego, CA., July 19-24, 1992.

2. J. B. Trenholme, "Overview of Basic Filamentation Concepts," Laser Modeling and Optimization talk, August 17, 1995; see also J. T. Hunt, "A Comparison of Nova and Beamlet's Design Margin Against Filamentation," LLNL memo NIF-LLNL-95-498, L-2037401 (1995).

3. W. H. Williams, K. R. Manes, J. T. Hunt, P. A. Renard, D. Milam, and D. Eimerl, "Modeling of Self-Focusing Experiments by Beam Propagation Codes," ICF Quarterly Report 6(1), Lawrence Livermore National Laboratory, Livermore, CA, UCRL-LR-105821-96-1 (1996), pp. 7-14.

4. P. L. Sulem, C. Sulem, and A. Patera, "Numerical Simulations of Singular Solutions to the Two-Dimensional Cubic Schrödinger Equation", Communications on Pure and Applied Mathematics, (John Wiley and Sons, New York), Vol. XXXVII, pp. 755-778.

5. M. D. Rotter on modeling pump-induced distortion on Beamlet (private communication).

7. S. N. Dixit, M. D. Feit, M. D. Perry and H. T. Powell, "Designing fully continuous phase screens for tailoring focal-plane irradiance profiles," Opt. Lett. 21 (1996) pp. 1715-1717; P. J. Wegner, B. M. VanWonterghem, C. E. Barker, J. A. Caird, S. N. Dixit, M. A. Henesian, J. T. Hunt, L. G. Seppala, C. E. Thomson, "Third-harmonic Performance of the Beamlet Prototype Laser," Second Annual International Conference on Solid State Lasers for Application to Inertial Confinement Fusion, Paris, France, October 22-25, 1996. 


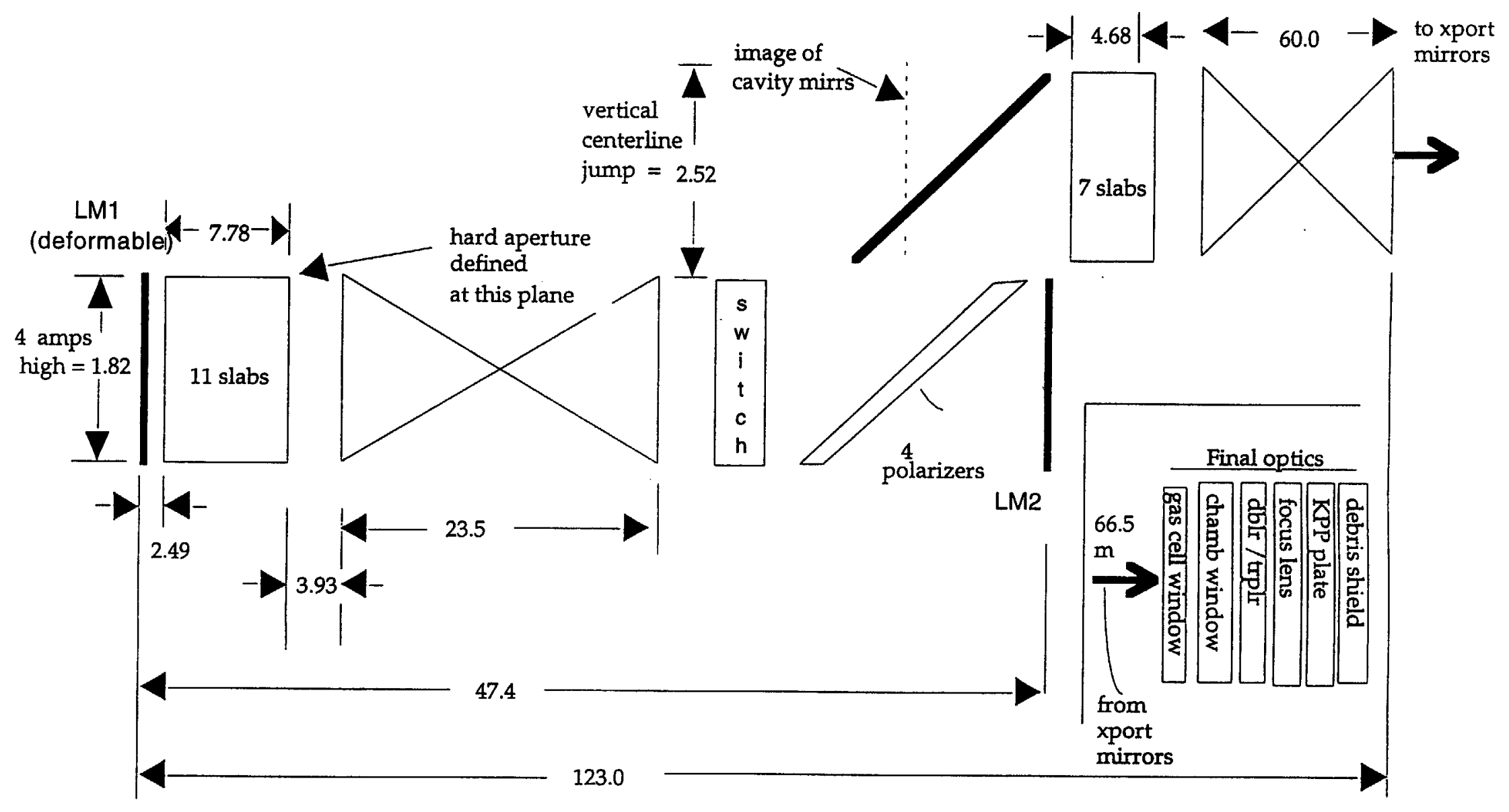

Figure 1. The NIF amplifier 11-0-7 architecture, as optimized using PROP92 in its 1D mode. 


\section{Interior slabs}

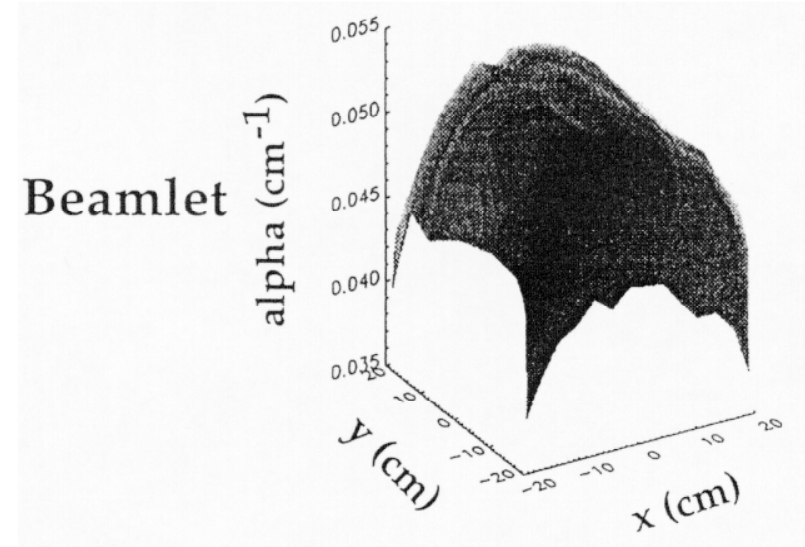

NIF

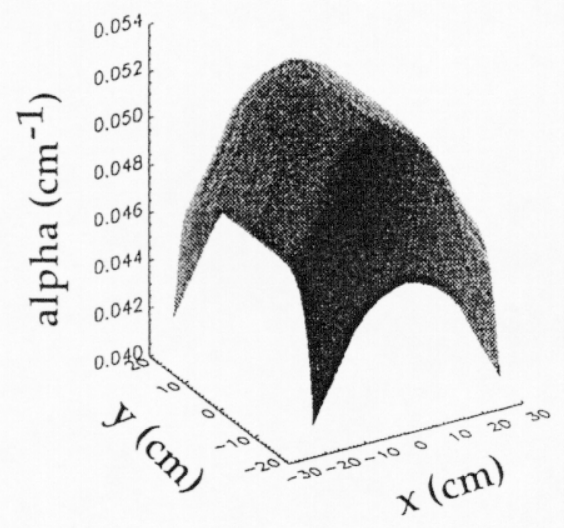

Exterior slabs
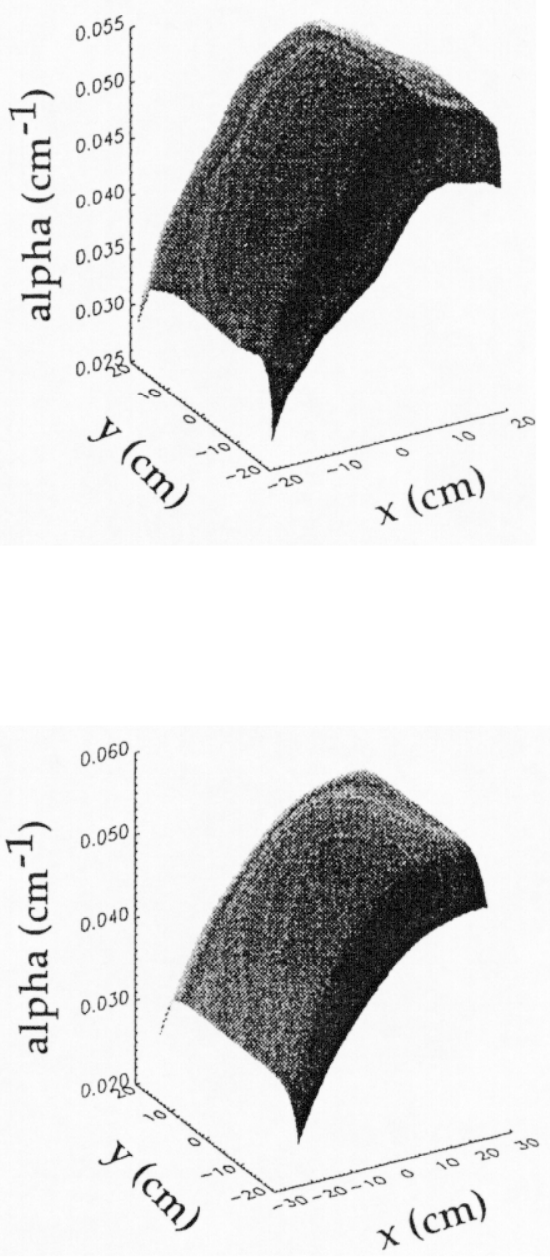

$0.04635 \mathrm{~cm}^{-1}$ $0.04491 \mathrm{~cm}^{-1}$

Figure 2. The assumed NIF transverse gain distribution is derived from Beamlet measurements. 

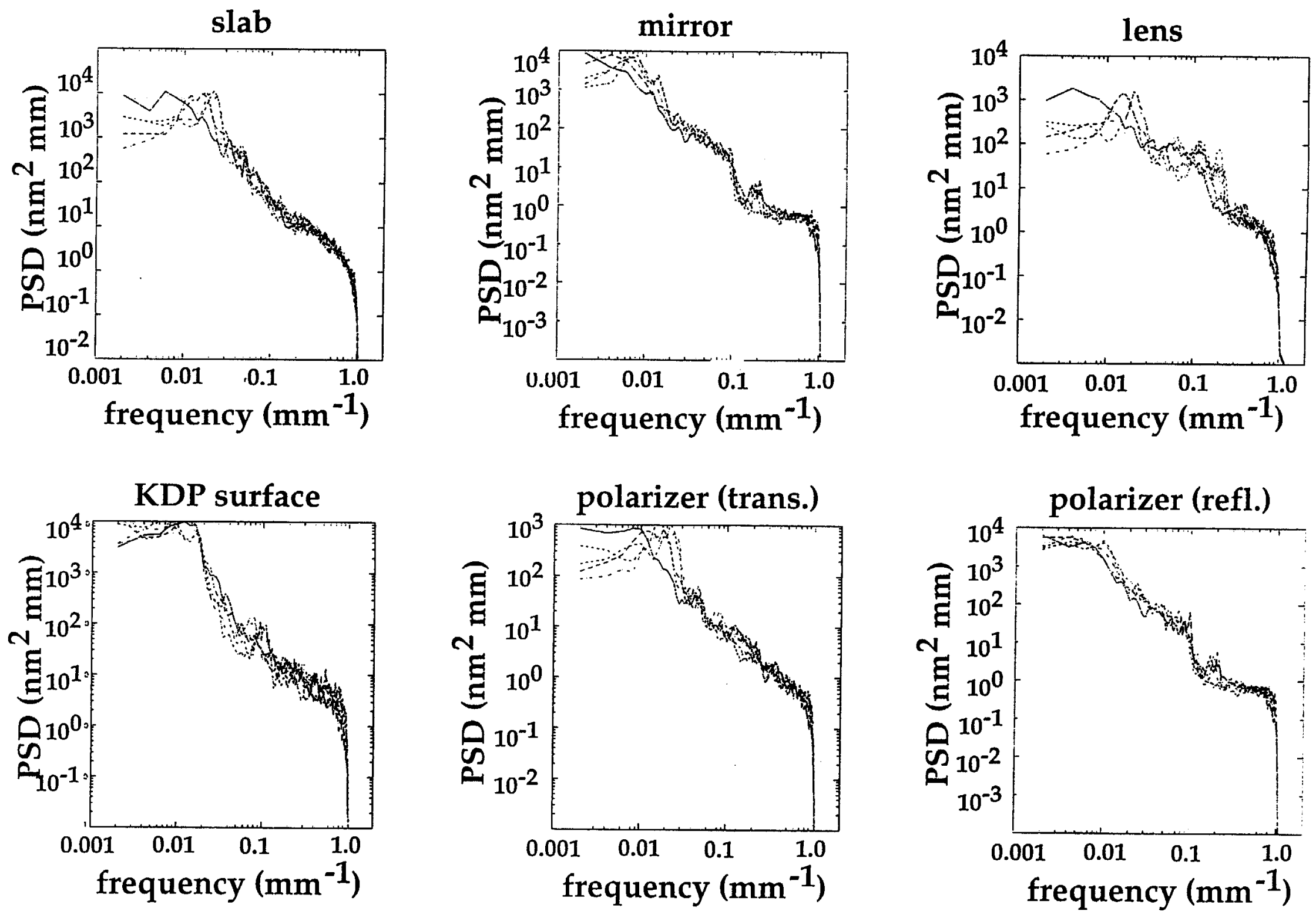

Figure 3. Measured PSD's for each type of optic in the chain. Independent phase screens are generated for each optic, matching the appropriate PSD. 


\section{slab phase screen}
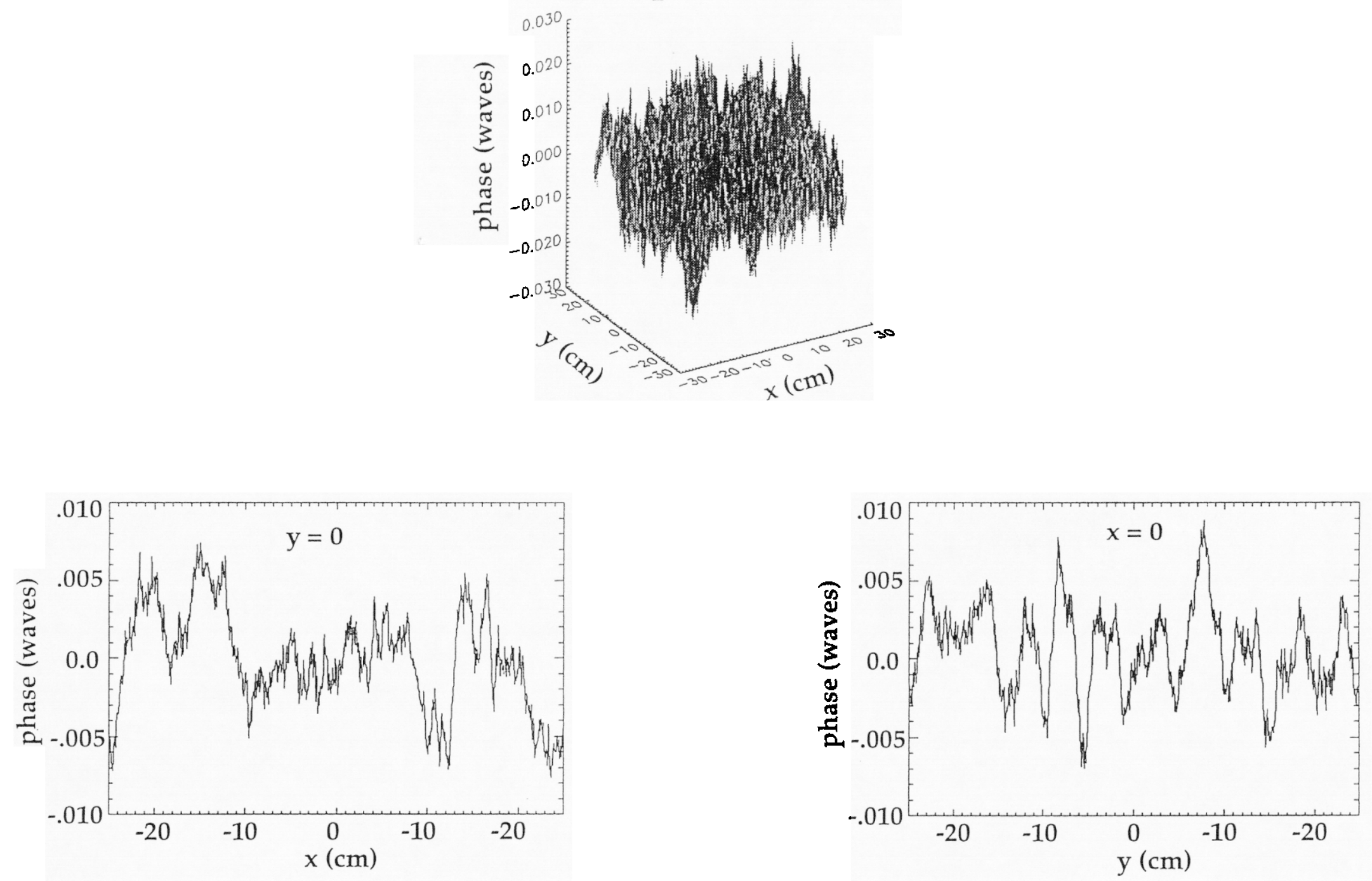

Fig. 4. Typical phase screen representing slab aberrations. 

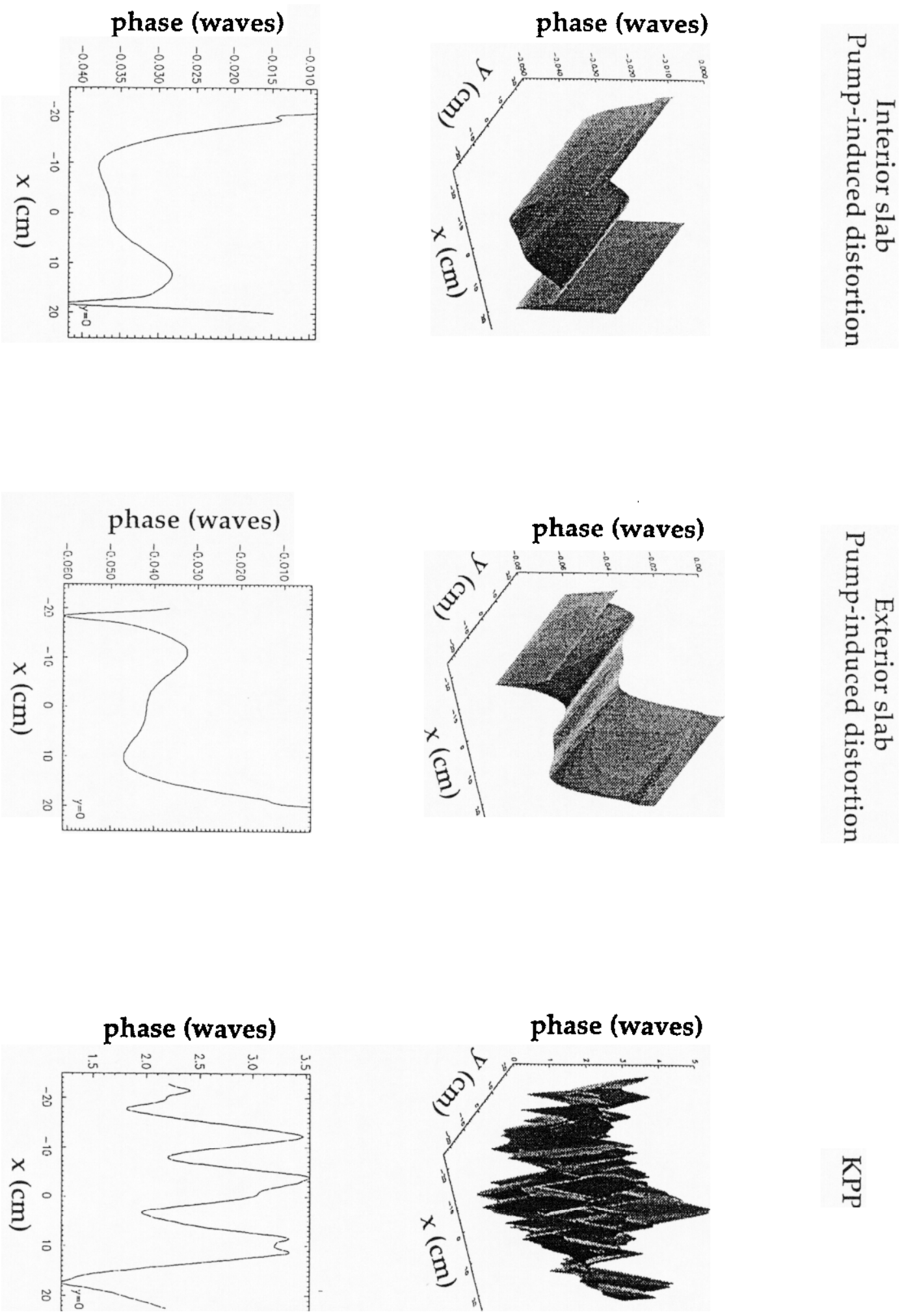

점 

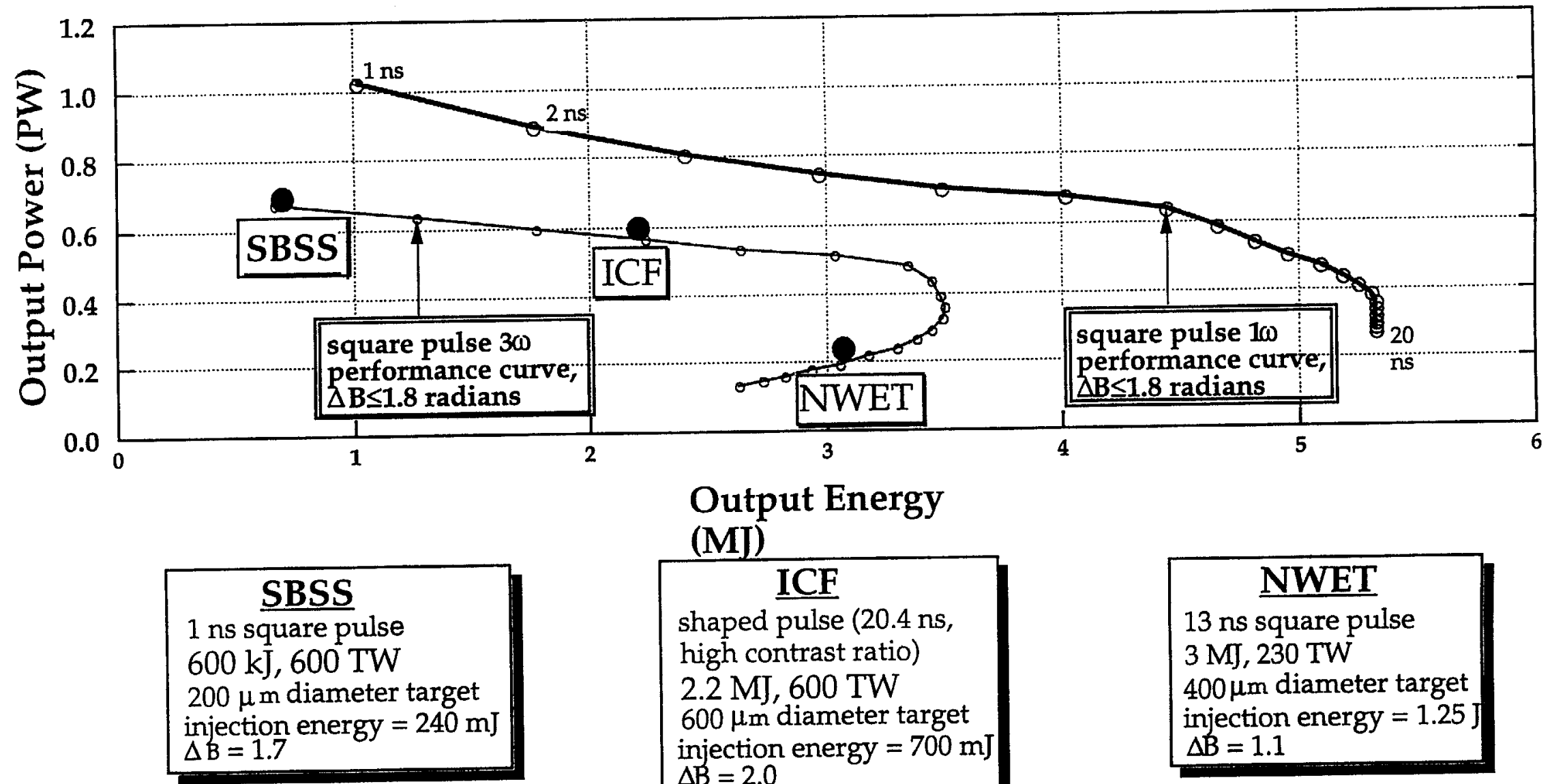

(MJ)
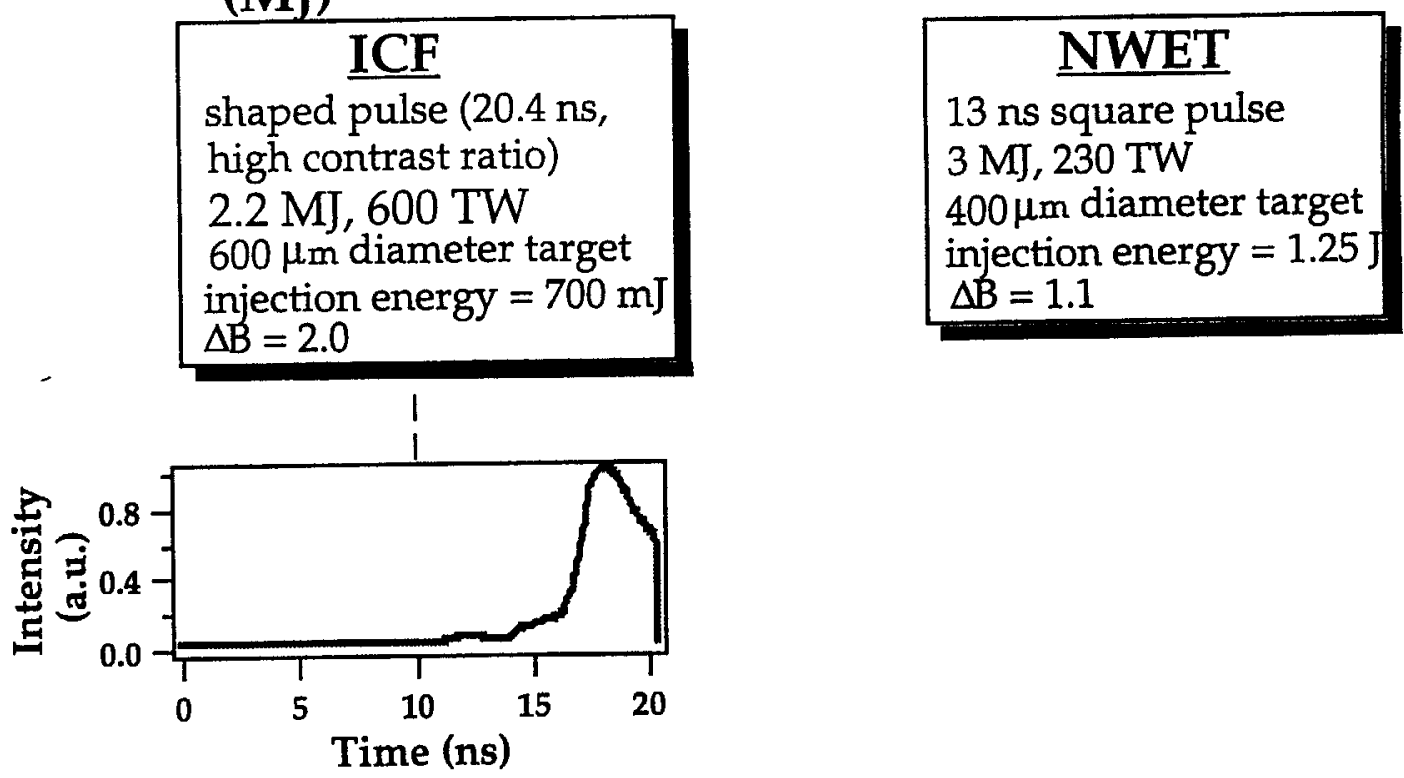

Fig. 6. The NIF baseline design meets the performance specifications for its three principle missions. 


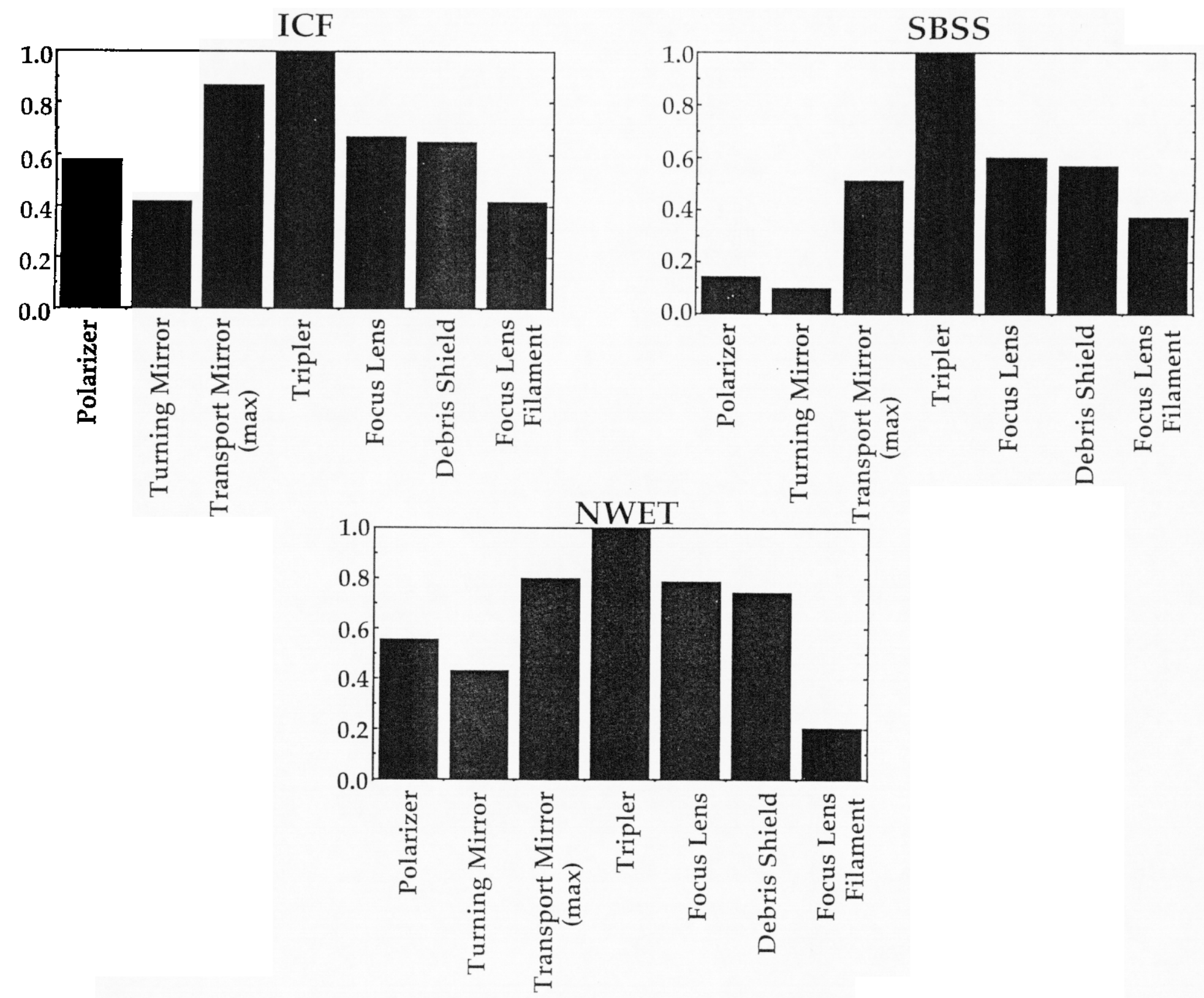

Fig. 7. In our model, NIF performance is limited by the $3 \omega$ damage threat to the tripler 

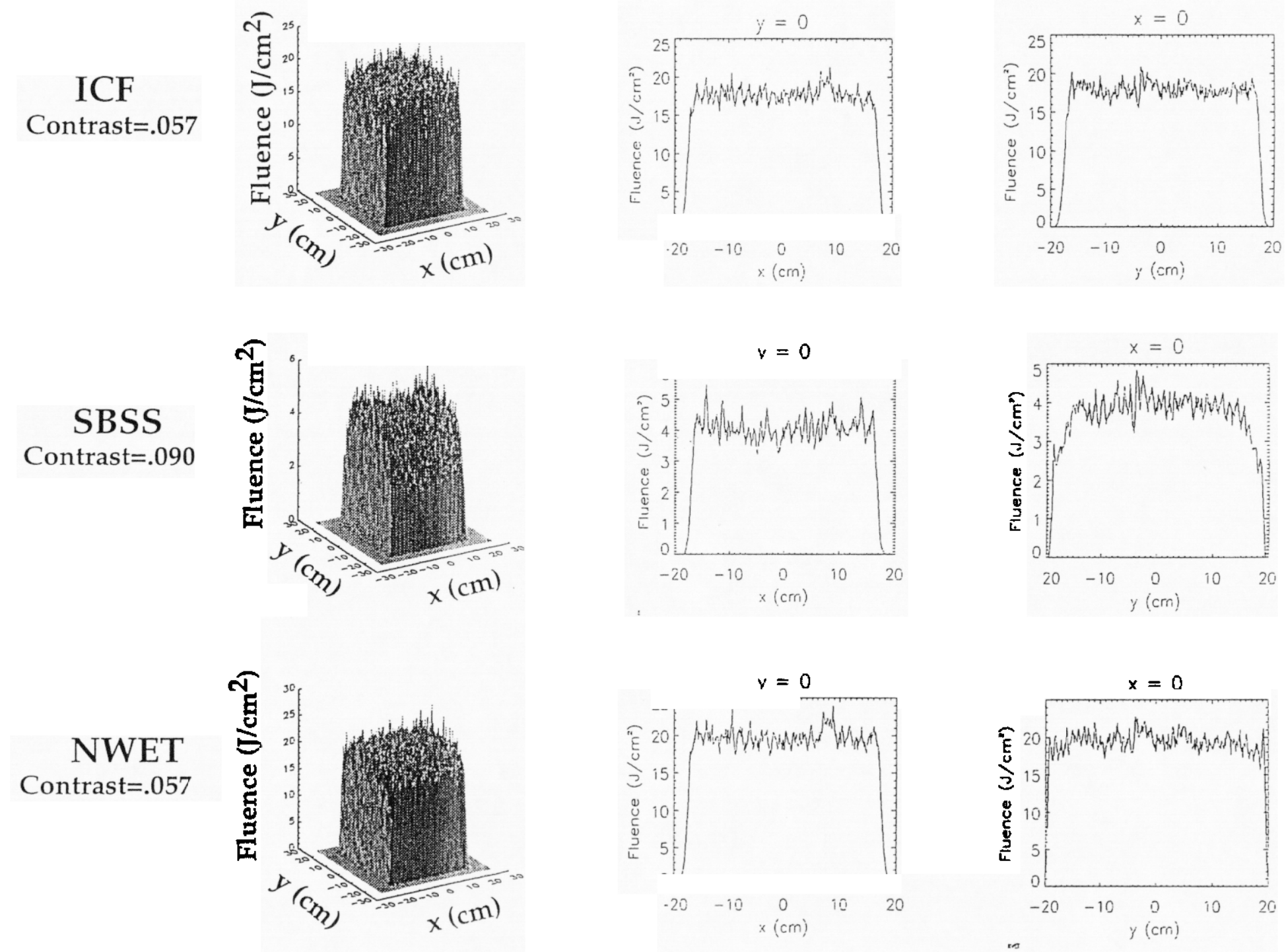

Figure 8. Near-field fluence modulation at the converter input is held to acceptable limits. 


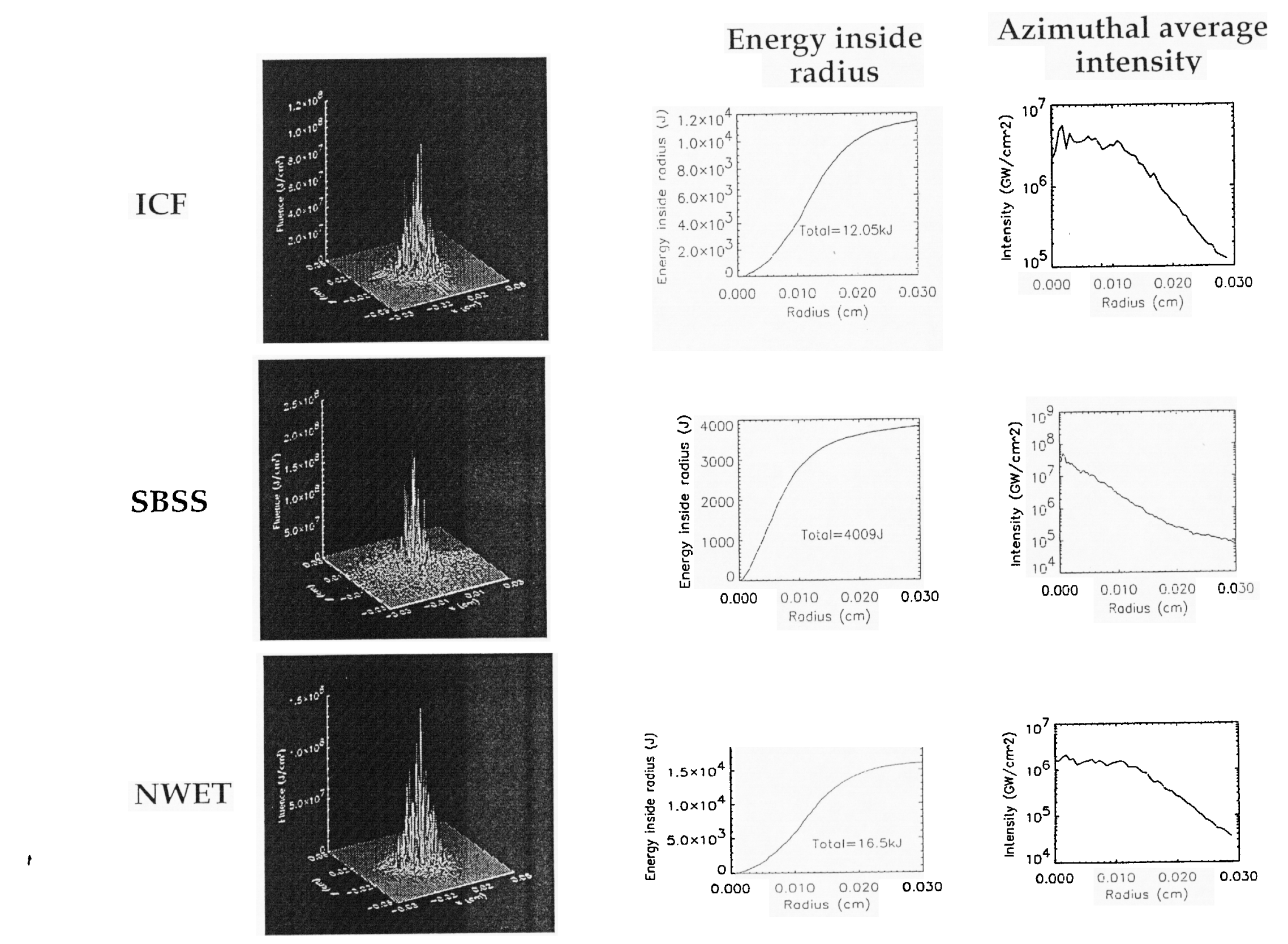

Fig. 9. NIF will deliver the required energy at the required target-plane spot size. 


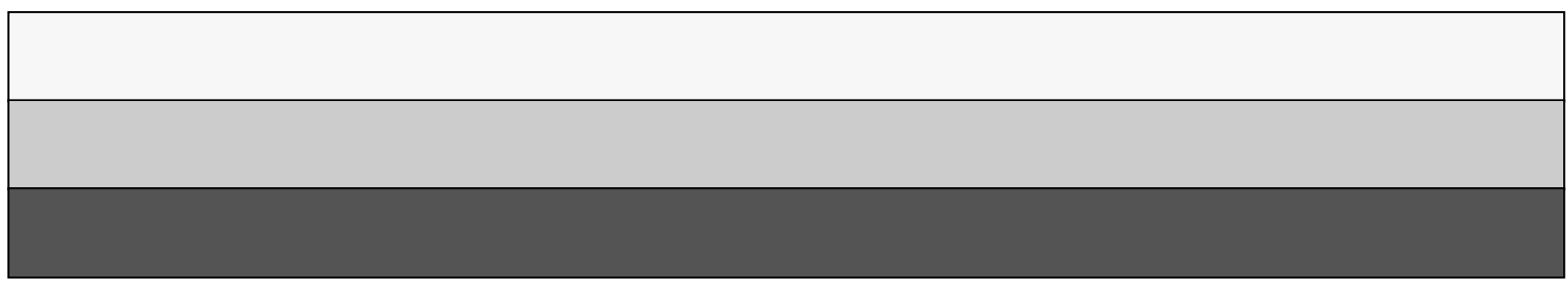

